\title{
Urinary reproductive hormones influence seed germination within diluted urine of heifers: alternative pregnancy diagnostic method
}

\author{
Iva Lázničková ${ }^{1}$, Tamara Fedorova $^{{ }^{*}}$, Magdaléna Štolcová ${ }^{2,3}$, Anna Kubátová ${ }^{1}$ \\ ${ }^{1}$ Department of Animal Science and Food Processing, Faculty of Tropical AgriSciences, Czech University of Life Sciences \\ Prague, Kamýcká 129, Prague - Suchdol 16500, Czech Republic \\ 2Department of Veterinary Sciences, Faculty of Agrobiology, Food and Natural Resources, Czech University of Life Sciences \\ Prague, Kamýcká 129, Prague - Suchdol 16500, Cžech Republic \\ ${ }^{3}$ Department of Cattle Breeding, Institute of Animal Science, Prátelstvi 815, Prague - Ubrinèves 10400, Cz̨ech Republic \\ *Corresponding author email:fedorova@ftz:czu.cz
}

Keywords: Germinated seeds, non-invasive pregnancy diagnosis, Triticum aestivum, Vigna radiata

Publication date 31/10/2020, http://m.elewa.org/Journals/about-japs/

\section{SUMMARY}

Experimental non-invasive pregnancy diagnostic techniques, such as seed germination testing in diluted urine, may serve as an alternative to standardized pregnancy diagnostic methods, but scientific validation of these methods is required. This study aimed to use Czech Fleckvieh heifers to investigate the influence of urinary reproductive hormones (oestrone sulphate, $17 \beta$-oestradiol, and pregnanediol3 -glucuronide) on the germination success of seeds placed within their diluted urine, and further to verify the reliability of seed germination test for pregnancy diagnosis in this species. Mung bean and wheat seeds were germinated in two urine-water dilutions (1:4 and 1:14) for three days, using urine samples obtained from either pregnant or non-pregnant heifers. Germinated seeds were counted daily, and the shoot lengths were measured three days after placement in the urine. Levels of urinary reproductive hormones were determined using competitive heterogeneous enzyme immunoassays (EIAs). Despite the dilution rate used, final results indicated that the urine from pregnant heifers inhibited germination and growth in mung beans only. On the contrary, wheat germination rate was higher within the urine from pregnant females on days 1 and 2, when using a 1:14 dilution rate. For both seed species, correlations between urinary hormone profiles and seed germination parameters were found, depending on the day of the experiment and the rate of dilution used. The shoot lengths of mung bean seeds were significantly $(\mathrm{P}<0.05)$ and negatively correlated with the profiles of all hormones analysed from the urine samples. Thus, urinary reproductive hormones influence seed germination and growth rate of wheat and mung beans, indicating the potential for this technique to be used for pregnancy determination in heifers.

\section{INTRODUCTION}

Pregnancy diagnosis is essential for better reproductive management in animals. To diagnose pregnancy, testing of urine samples obtained non-invasively by free catch without the animal being restrained or sedated can be a suitable option in different species (Kubátová et al., 2016). For the purpose of pregnancy diagnosis, urine samples can be processed by reproductive hormone assays (Kirkpatrick et al., 1992) or experimental diagnostic methods, such 
as the seed germination test (Narayana Swamy et al., 2010). The seed germination test compares shoot length and the number of germinated seeds in diluted urine of pregnant and non-pregnant females (Rine et al., 2014). According to previous studies, the urine of pregnant cows significantly inhibits the germination and growth of seeds (Veena and Narendranath, 1993; Dilrukshi and Perera, 2009; Skálová et al., 2017). However, factors influencing the differences in the germination of seeds in urine of pregnant and non-pregnant animals are still unknown. To the best of the knowledge of the authors of this study, no studies have reported the relation between urinary reproductive hormones in cattle and the germination of seeds kept in diluted urine. To date, only one study has examined the effects of different concentrations of 17Boestradiol (E2) and progesterone (P4) on mung bean and wheat seeds and concluded that they do not influence seed germination rate (Nirmala et al., 2008). The determination of reproductive hormones in the urine by enzyme-immunoassay (EIA) has been described in several ungulate species (Kirkpatrick et al., 1991; Volkery et al., 2012). Pregnanediol-3-glucuronide (PdG) is a urinary metabolite of P4 (Loskutoff et al., 1983)

\section{MATERIALS AND METHODS}

The research was carried out in accordance with the current legislation of the Czech Republic and the European Union and was approved by the management of the farm involved, i.e. dairy farm in Vendolí. According to Act no. 246/1992 Coll. of the Czech Republic and Directive 2010/63/EU of the European Parliament and of the Council of 22 September 2010 on the protection of animals used for scientific purposes, the study met all of the requirements of non-invasive research so no other ethical approval was needed. Animals were familiarized gradually to the sampling devices and sampling process and they were not forced to cooperate to prevent their stress. and was found at higher concentrations in pregnant cows than in non-pregnant ones with a peak on day 16 after artificial insemination (Yang et al., 2004). While P4 remains at constant levels during cattle gestation and decreases approximately 10 days before parturition (Catchpole, 1969), the concentrations of plasma oestrone sulphate (E1S) and E2 increase progressively as pregnancy advances and reach peak levels before calving (Desaulniers et al., 1989; Shah et al., 2006). The concentration of E1S increases earlier in the urine than in the blood of pregnant cows (Yang et al., 2003). This study investigated the influence of urinary reproductive hormones on the germination of seeds kept in diluted urine of cattle, which can serve as a model species for related wildlife such as buffaloes or antelopes. Moreover, it aimed to identify differences in seed germination rate in the urine of pregnant and non-pregnant heifers for possible pregnancy diagnosis. The results may help in implementing the seed germination test for better pregnancy diagnosis in small farms or breeding facilities where sophisticated laboratory equipment is not available or its use is limited due to limited possibilities of handling untamed animals.

3.1 Animals: Czech Fleckvieh heifers ( $\mathrm{n}=$ 12 pregnant, $n=10$ non-pregnant) from a dairy farm in Vendolí, the Czech Republic $\left(49^{\circ} 44^{\prime} 10^{\prime \prime} \mathrm{N} 16^{\circ} 24^{\prime} 45^{\prime \prime} \mathrm{E}, 480\right.$ MASL), were randomly selected for the study. The heifers were fed with a total mixed ration composed of clover haylage, corn silage, straw, grain, and mineral supplements for heifers. All heifers were free of reproductive and digestive disorders. Females were considered as pregnant when a veterinarian using transrectal ultrasonography (at the earliest 28 days after artificial insemination) confirmed their pregnancy. All pregnancies were verified by a successful parturition as well. Non-pregnant heifers served 
as control animals, and their oestrous cycles were not monitored.

3.2 Urine sample collection: The research was carried out from May to December 2014. The urine of pregnant heifers was non-invasively collected at 2 -week intervals from the $4^{\text {th }}$ to $8^{\text {th }}$ week of pregnancy. The urine of non-pregnant heifers was sampled at irregular intervals. Urine collection started after morning feeding, around 06:00 AM. Midstream urine was collected into half-litre plastic cups fastened on a telescopic rod during spontaneous urination (Haberová et al., 2011). For the seed germination tests, urine was poured into closable plastic vials (20 or 60 $\mathrm{ml}$ ), refrigerated at $5-7^{\circ} \mathrm{C}$, and transported to the laboratory within 12 hours (Haberová et al., 2011). For the hormonal assays, urine was poured into three closable plastic Eppendorf tubes $\left(3\right.$ or $5 \mathrm{ml}$ ), frozen at $-20^{\circ} \mathrm{C}$ (Volkery et al., 2012) and transported within 12 hours to the laboratory in a car refrigerator surrounded by frozen gel to prevent the samples from defrosting. The samples were stored at $-20{ }^{\circ} \mathrm{C}$ until hormone analyses (Volkery et al., 2012).

\subsection{Sample preparation for seed} germination test: The germination tests were performed by diluting urine samples with distilled water at the ratio of 1:4 (1 part urine and 4 parts water) and 1:14 (1 part urine and 14 parts water) (Dilrukshi and Perera, 2009; Narayana Swamy et al., 2010). Winter wheat (Triticum aestivum) and mung bean (Vigna radiata) seeds intended for human consumption and bought in a grocery store were used for this research. Seeds were prepared in sterile Petri dishes and the urine-water solution $(20 \mathrm{ml})$ was applied onto the seeds. Four seed germination tests, with 50 seeds in each sterile Petri dish, were performed for every urine sample: 1) mung beans treated with dilution $1: 4 ; 2$ ) mung beans treated with dilution $1: 14$; 3) wheat seeds treated with dilution $1: 4$; 4) wheat seeds treated with dilution 1:14. The prepared tests containing seeds together with the urine-water solution were kept under laboratory conditions for 3 consecutive days at a constant room temperature of around $25^{\circ} \mathrm{C}$ under natural day light. The number of germinated seeds was counted daily, always at the same time of day, and the length of shoots was measured at the end of the test (day 3) using a ruler. Control experiments were carried out with $20 \mathrm{ml}$ of distilled water and 50 mung bean or 50 wheat seeds in each Petri dish (Veena and Narendranath, 1993; Narayana Swamy et al., 2010) under the same conditions.

3.4 Urinary hormone assays: The urinary reproductive hormones (E1S, E2 and PdG) were determined by competitive heterogeneous enzyme immunoassay (EIA). The Estrone Enzyme Immunoassay Kit, 17- $\beta$-oestradiol Enzyme Immunoassay Kit and Pregnanediol-3Glucuronide Enzyme Immunoassay (Arbor Assays, USA) were used. Sample preparation, reagent preparation and the assay protocol were performed according to the manufacturer's recommendations (Detect $X^{\circledR}$, Arbor Assays, USA). To determine the optimal concentration of diluted urine to use for the assay, three dilution factors of tested samples were compared to a standard curve generated using positive control hormones supplied with the assay. Urine diluted with assay buffer (component of assay kit) was tested before the final urine hormone assay. Three dilution factors were prepared for each tested hormone to achieve the ideal dilutions, which were equivalent to the standards of each particular hormone. The dilution that was equivalent to the standards of a particular hormone (determined by Arbor Assays, USA) was used in the subsequent urine hormone examination. Standards were prepared by serial dilution of standard solution (i.e. solution with known concentration of given hormone). The final dilutions used for urinary E1S, E2 and PdG determination are displayed in Table 1.

Table 1: Dilution of urine samples with Assay Buffer 


\begin{tabular}{l|l|l}
\hline Hormone & Pregnancy status of heifers & Urine sample: Assay Buffer \\
\hline \multirow{2}{*}{ Oestrone sulphate } & Pregnant & $1: 12$ \\
\cline { 2 - 3 } & Non-pregnant & $1: 4$ \\
\hline \multirow{2}{*}{$17 \beta$-oestradiol } & Pregnant & $1: 1$ \\
\cline { 2 - 3 } & Non-pregnant & $1: 1$ \\
\hline \multirow{2}{*}{ Pregnanediol-3-glucuronide } & Pregnant & $1: 9$ \\
\cline { 2 - 3 } & Non-pregnant & $1: 4$ \\
\hline
\end{tabular}

The hormone concentrations were evaluated by measuring absorbance with a spectrophotometer (VersaMax ELISA reader, Molecular Devices, USA) at wavelength $\lambda=450$ nm. A four-parameter logistic calibration curve was generated using standard samples. The concentrations of the hormones in the urine samples were determined from the standard curve using SoftMax Pro 5 software (Molecular Devices, USA).

3.5 Data analysis: Data were analysed using Statistica Cz 12 software (StatSoft, Inc., 2013). As the data for the number of germinated seeds did not show a normal distribution, the influence

\section{RESULTS}

Totally 36 and 50 urine samples were obtained non-invasively from pregnant and non-pregnant heifers, respectively. In total, 8,600 mung beans and 8,600 wheat seeds were processed in the seed germination tests, among which 4,844 mung beans and 1,929 wheat seeds germinated.

4.1 Number of germinated seeds: The presence of heifers' urine, regardless of pregnancy status, inhibited the number of mung bean and wheat seeds that germinated in both dilutions compared to the control conditions containing distilled water for all three days of the test $(\mathrm{P}<0.01$ for all cases). Pregnancy status did not significantly $(\mathrm{P}>0.05)$ influence the number of mung bean seeds that germinated in both dilutions on days 1 and 2 of the experiment. On day 3 , a significantly lower number of germinated mung bean seeds was counted in urine obtained from pregnant heifers than in non-pregnant heifers for both dilutions $(\mathrm{P}=$ 0.020 and $\mathrm{P}=0.015$ for dilution $1: 4$ and $1: 14$, of pregnancy on the number of germinated seeds was statistically evaluated using the nonparametric Mann-Whitney $U$ test. The influence of pregnancy on the length of shoots was evaluated by ANOVA. The differences between concentrations of reproductive hormones in pregnant and non-pregnant heifers were evaluated using the Mann-Whitney U test. The relationship between reproductive hormones and the number of germinated seeds as well as the lengths of shoots was tested using the Spearman's rank correlation. Significance was accepted at 0.05 .

respectively). The results for wheat seeds showed the opposite trend. No significant differences were observed in the number of wheat seeds that germinated in urine of pregnant and non-pregnant heifers when exposed to 1:4 dilution ( $\mathrm{P}>0.05$ for all three days) whereas significant differences were observed when wheat seeds were exposed to a 1:14 dilution on day $1(\mathrm{P}=0.001)$ and day $2(\mathrm{P}=0.031)$ of the experiment. For detailed results see Table 2.

4.2 Length of shoots: The length of shoots was also inhibited by the presence of heifers' urine, regardless of pregnancy status. Significantly shorter shoots were measured in mung bean seeds that germinated in urine-water solutions $(\mathrm{P}<0.001$ for both dilutions $)$ compared to distilled water only. Similar findings were observed in wheat seeds $(\mathrm{P}<0.001$ and $\mathrm{P}$ $<0.01$ for dilution 1:4 and 1:14, respectively). Significantly shorter shoots were measured in mung bean seeds that germinated in the urine of 
pregnant heifers ( $\mathrm{P}<0.0001$ for both dilutions). No significant effect $(\mathrm{P}>0.05)$ of pregnancy status on shoot length was observed in wheat at either dilution of urine.

Table 2: Mean number of germinated mung beans and wheat seeds kept in urine diluted 1:4 and 1:14 during all three days of the test.

\begin{tabular}{|c|c|c|c|c|c|c|}
\hline \multirow[t]{2}{*}{ Test } & \multirow[t]{2}{*}{ Day } & \multicolumn{2}{|c|}{ Pregnant heifers } & \multicolumn{2}{|c|}{$\begin{array}{l}\text { Non-pregnant } \\
\text { heifers }\end{array}$} & \multirow[t]{2}{*}{$\begin{array}{l}\text { Significance } \\
\text { level }\end{array}$} \\
\hline & & Mean & SE & Mean & SE & \\
\hline \multirow{3}{*}{$\begin{array}{l}\text { Mung beans dilution } \\
1: 4\end{array}$} & 1 & 12.944 & 1.632 & 13.040 & 1.173 & $\mathrm{P}>0.05$ \\
\hline & 2 & 15.389 & 1.687 & 19.520 & 1.674 & $\mathrm{P}>0.05$ \\
\hline & 3 & 16.528 & 1.815 & 22.540 & 1.788 & $\mathrm{P}<0.05$ \\
\hline \multirow{3}{*}{$\begin{array}{l}\text { Mung beans dilution } \\
1: 14\end{array}$} & 1 & 25.028 & 1.823 & 21.980 & 1.631 & $\mathrm{P}>0.05$ \\
\hline & 2 & 30.306 & 1.582 & 32.940 & 1.710 & $\mathrm{P}>0.05$ \\
\hline & 3 & 32.639 & 1.667 & 37.320 & 1.727 & $\mathrm{P}<0.05$ \\
\hline \multirow{3}{*}{$\begin{array}{l}\text { Wheat seeds dilution } \\
1: 4\end{array}$} & 1 & 7.250 & 0.798 & 5.480 & 0.533 & $\mathrm{P}>0.05$ \\
\hline & 2 & 8.278 & 0.862 & 6.700 & 0.582 & $\mathrm{P}>0.05$ \\
\hline & 3 & 8.556 & 0.889 & 7.180 & 0.628 & $\mathrm{P}>0.05$ \\
\hline \multirow{3}{*}{$\begin{array}{l}\text { Wheat seeds dilution } \\
1: 14\end{array}$} & 1 & 14.306 & 1.087 & 10.160 & 0.589 & $\mathrm{P}<0.01$ \\
\hline & 2 & 15.333 & 1.093 & 12.520 & 0.764 & $\mathrm{P}<0.05$ \\
\hline & 3 & 15.778 & 1.084 & 13.880 & 0.906 & $\mathrm{P}>0.05$ \\
\hline
\end{tabular}

Significance level indicates statistical differences between pregnant and non-pregnant heifers.

4.3 Hormonal profiles and their pregnant heifers are displayed in Table 3. All relationships to seed germination rate and shoot length: The mean concentrations of results differed significantly between pregnant and non-pregnant heifers $(\mathrm{P}<0.001)$. urinary E1S, E2 and PdG in pregnant and non-

Table 3: Mean \pm SE concentrations of urinary oestrone sulphate, $17 \beta$-oestradiol and pregnanediol3-glucuronide.

\begin{tabular}{l|c|c}
\hline Hormone & Pregnant heifers & Non-pregnant heifers \\
\hline Oestrone sulphate $(\mathrm{pg} / \mathrm{ml})$ & $2477.186 \pm 107.826$ & $808.929 \pm 59.871$ \\
\hline $\begin{array}{l}17 \beta \text {-oestradiol }(\mathrm{ng} / \mathrm{ml}) \\
\begin{array}{l}\text { Pregnanediol-3-glucuronide } \\
\text { (ng/ml) }\end{array}\end{array}$ & $225.019 \pm 0.830$ & $2.505 \pm 0.180$ \\
\hline All & & $26.650 \pm 1.683$ \\
\hline
\end{tabular}

All results differed significantly between pregnant and non-pregnant heifers $(\mathrm{P}<0.001)$. 
The detailed correlations between measured concentrations of reproductive hormones and

numbers of germinated seeds are displayed in Table 4.

Table 4: The correlations between concentrations of reproductive hormones and numbers of germinated seeds.

\begin{tabular}{l|l|l|l|l}
\hline \multirow{2}{*}{ Test } & Day & $\begin{array}{l}\text { Oestrone } \\
\text { sulphate }\end{array}$ & $\mathbf{1 7 \beta}$-oestradiol & $\begin{array}{l}\text { Pregnanediol-3- } \\
\text { glucuronide }\end{array}$ \\
\cline { 2 - 5 } & & $r$ & $r$ & $R$ \\
\hline \multirow{3}{*}{ Mung beans dilution 1:4 } & 1 & 0.005 & -0.107 & 0.061 \\
\cline { 2 - 5 } & 2 & -0.207 & $-0.241^{*}$ & -0.146 \\
\cline { 2 - 5 } & 3 & $-0.269^{*}$ & $-0.284^{*}$ & -0.210 \\
\hline \multirow{2}{*}{ Mung beans dilution 1:14 } & 1 & 0.086 & -0.080 & 0.053 \\
\cline { 2 - 5 } & 2 & -0.140 & $-0.321^{*}$ & $-0.227^{*}$ \\
\cline { 2 - 5 } & 3 & $-0.235^{*}$ & $-0.361^{*}$ & $-0.352^{*}$ \\
\hline \multirow{2}{*}{ Wheat seeds dilution 1:4 } & 1 & 0.096 & 0.125 & $0.339^{*}$ \\
\cline { 2 - 5 } & 2 & 0.038 & 0.088 & $0.286^{*}$ \\
\cline { 2 - 5 } & 3 & 0.05 & 0.093 & $0.296^{*}$ \\
\hline \multirow{2}{*}{ Wheat seeds dilution 1:14 } & 1 & $0.242^{*}$ & $0.416^{*}$ & $0.381^{*}$ \\
\cline { 2 - 5 } & 2 & 0.132 & $0.372^{*}$ & $0.251^{*}$ \\
\cline { 2 - 5 } & 3 & 0.043 & $0.294^{*}$ & 0.175 \\
\hline
\end{tabular}

Legend: * Significant correlation at $\mathrm{P}<0.05$ level.

Weak but significant $(\mathrm{P}<0.05)$ negative correlations were found between the length of mung bean shoots and concentrations of all measured reproductive hormones both for dilution 1:4 $(r=-0.139, r=-0.111$, and $r=-$ 0.125 for E1S, E2 and PdG, respectively) and for dilution 1:14 $(r=-0.190, r=-0.269$ and $r=$ 0.274 for E1S, E2 and PdG, respectively). In the

\section{DISCUSSION}

5.1 Seed germination test: No matter the type of seed, the control test results were consistent with the results of other authors who confirmed better seed germination rate in distilled water compared to urine-water solutions (Veena and Narendranath, 1993; Dilrukshi and Perera, 2009; Kubátová and Fedorova, 2016). In this study, significant differences were observed in the number of mung bean seeds that had germinated after 3 days, which is consistent with the results of previous studies (Narayana Swamy et al., 2010; Rine et al., 2014). The same authors (Narayana case of wheat seeds treated with dilution 1:4, there were significant $(\mathrm{P}<0.05)$ weak positive correlations between the length of shoots and E1S $(r=0.086)$ and PdG $(r=0.214)$. However, there was no significant correlation $(\mathrm{P}>0.05)$ between the length of wheat shoots treated with dilution 1:14 and all measured reproductive hormones.

Swamy et al., 2010; Rine et al., 2014) measured the lengths of shoots on day 5 of a germination test, but according to the results obtained by this study, significant differences are readily visible after only 3 days, which is consistent with a study in female mithun (Bos frontalis) using paddy seeds (Perumal, 2014). The results from day 3 concerning germinated mung bean seeds and the lengths of their shoots are consistent with studies that reported an inhibitory effect of cows' pregnancy on mung bean germination (Dilrukshi and Perera, 2009; Rao Krishna and Veena, 2009; Skálová et al., 2017). Surprisingly, 
the results of this study for wheat seeds germinating in the dilution 1:14 during days 1 and 2 correspond with those of Ghalioungui et al. (1963) when diagnosing pregnancy in humans. However, in cattle, the opposite trend would be expected (Veena and Narendranath, 1993), as was observed in mung beans. Nevertheless, with the exception of these two days in the 1:14 dilution, no differences were found in either seed germination rate or shoot lengths of wheat seeds in the urine of pregnant and non-pregnant heifers. Thus, it can be assumed that the different varieties of wheat used might have different seedling emergence characteristics (Mohan et al., 2013). Irrespective of the fact that seed germination rate is affected by different factors such as moisture, temperature, daylight, nutrition or seed storage and that different plant species have different requirements (White and Edwards, 2007), $20 \mathrm{ml}$ of urine-water solution were applied to both kinds of seeds. While mung bean seeds almost depleted the whole solution due to significant swelling during their germination, wheat seeds were under the surface of the solution for the entire experimental period. In addition, darkening of the seeds, colour changes in the urine-water solution or mildew in the Petri dishes that contained wheat seeds was observed. It is possible that these factors influenced the results and precluded the finding of any differences at the end of the experiment.

\subsection{Reproductive hormone assays:} Urinary hormone levels are generally calibrated to creatinine values in urine (Kirkpatrick et al., 1991; Yang et al., 2003). However, normalizing against urinary creatinine was not needed for the purpose of this study because the exact concentrations of hormones were required to study their relationship with seed germination rate. The results of this study for urinary E2 profiles in pregnant and non-pregnant heifers are consistent with studies carried out on E2 profiles in plasma (Robertson and King, 1979; Patel et al., 1999). E2 concentrations increased as pregnancy advanced, and higher E2 concentrations were confirmed in pregnant females. Based on the results for urinary PdG as well as those from Yang et al. (2004), urinary PdG monitoring can be considered a useful diagnostic method for pregnancy in heifers. Yang et al. (2004) reported three- to four-fold higher $\mathrm{PdG}$ concentrations in the urine of pregnant cows 21 days after AI. However, these results were obtained from only two pregnant cows. Nevertheless, the results of this study support these findings as they showed approximately eight times higher mean urinary PdG concentrations in heifers that were 4-8 weeks pregnant compared to non-pregnant heifers. Kirkpatrick et al. (1991; 1992) also reported higher PdG concentrations in the urine of pregnant bison cows (Bison bison).

\subsection{Interaction of reproductive urinary} hormones and seed germination rate: Although Nirmala et al. (2008) determined that E2 and P4 are not efficient inhibiting/inducing factors of germination and growth, this study found weak correlations between urinary reproductive hormone profiles and the germination and growth of seeds. Notably, it was observed that urinary E2 and PdG correlated more frequently with seed germination rate and shoot growth than E1S. Moreover, the influence of E1S and PdG on seed germination rate has never been tested. It can be assumed that mung beans germinated and grew less under the influence of higher urinary PdG concentrations. Although some studies suggest that higher concentrations of $\mathrm{ABA}$ in the urine of pregnant female cattle result in seed dormancy by inhibiting germination (Veena Ganesaiah, 2006; Dilrukshi and Perera, 2009), the results of this study demonstrate a different response of mung beans and wheat seeds to the urine of pregnant female heifers. Thus, the authors of this study agree with the statement of Rao Krishna and Veena (2009) that “...plant growth regulators such as auxins and abscisic acid are likely to be excreted in urine as and when the animals 
consume plants containing such substances" and conclude that $\mathrm{PdG}$ is likely the main factor influencing germination, rather than $\mathrm{ABA}$. Hence, the results of this study confirm that the urine of pregnant heifers inhibits mung bean seeds germination and growth as evidenced by lower germination rates and shorter shoots. The most obvious effect on the germination of wheat seeds was observed in a 1:14 dilution and displayed the opposite trend. Pregnancy status did not influence the length of wheat seed shoots. Higher concentrations of E1S, E2 and PdG were observed in the urine of pregnant heifers than in non-pregnant ones. It can be hypothesized that higher oestrogens and PdG concentrations in the urine of pregnant heifers

\section{ACKNOWLEDGMENTS}

The research and article production were financed by the Internal Grant Agency of the Faculty of Tropical AgriSciences CZU Prague (project no. 20205005) and the Czech University of Life Sciences Prague University Internal Grant Agency (projects no. 20145009 and

\section{REFERENCES}

Catchpole HR: 1969. Hormonal mechanisms during pregnancy and parturition. P.417440. In: Reproduction in domestic animals. Cole HH. and Cupps PT (Editors), Academic Press, New York.

Desaulniers DM, Goff AK, Betteridge KJ, Rowell JE. and Flood PF: 1989. Reproductive hormone concentrations in faeces during the oestrous cycle and pregnancy in cattle (Bos taurus) and muskoxen (Ovibos moschatus). Canadian Journal of Zoology 67: 1148-1154.

Dilrukshi HNN. and Perera ANF: 2009. Evaluation of an ancient technique to diagnose the pregnancy in cattle using urine. Wayamba Journal of Animal Science 1: 6-8.

Ghalioungui P, Khalil S. and Ammar AR: 1963. On an ancient Egyptian method of can inhibit mung bean germination rate as well as shoot growth. Based on the results of this study, mung beans rather than wheat seeds can be recommended for the seed germination test because of better visibility of seedling emergence and clearer results compared with those obtained using wheat seeds. When sophisticated laboratory equipment or financial support is available, using hormonal assays for time efficiency, reliability, and consistency is recommended. Specifically, urinary $\mathrm{PdG}$ measurements can be regarded as a suitable method for early pregnancy diagnosis in heifers. Otherwise, a non-invasively processed seed germination test could serve as a urine-based alternative

20175006). We thank the breeders from the farm Vendolí for their cooperation. Special thanks belong to prof. Eva Baranyiová for her professional leadership and editorial contribution.

diagnosing pregnancy and determining foetal sex. Medical History 7: 241-246.

Haberová T, Koláčková K. and Lukešová D: 2011. Pregnancy diagnosis by chemical tests of urine in camels (Camelus bactrianus). p.76. In: $8^{\text {th }}$ International Conference on Behaviour, Physiology and Genetics of Wildlife, Berlin.

Kirkpatrick JF, Bancroft K. and Kincy V: 1992. Pregnancy and ovulation detection in bison (Bison bison) assessed by means of urinary and fecal steroids. Journal of Wildlife Diseases 28: 590-597.

Kirkpatrick JF, Kincy V, Bancroft K, Shideler SE. and Lasley BL: 1991. Oestrous cycle of the North American bison (Bison bison) characterized by urinary pregnanediol-3glucuronide. Journal of Reproduction and Fertility 93: 541-547. 
Kubátová A. and Fedorova T: 2016. Seed germination test as an alternative urinebased non-invasive pregnancy test in alpacas (Vicugna pacos). Journal of Camel Practice and Research 23: 261-264.

Kubátová A, Fedorova T, Skálová I. and Hyniová L: 2016. Non-invasive pregnancy diagnosis from urine by the Cuboni reaction and the barium chloride test in donkeys (Equus asinus) and alpacas (Vicugna pacos). Polish Journal of Veterinary Sciences 19: 477-484.

Loskutoff NM, Ott JE. and Lasley BL: 1983. Strategies for assessing ovarian function in exotic species. Journal of Zoo Animal Medicine 14: 3-12.

Mohan A, Schillinger WF. and Gill KS: 2013. Wheat Seedling Emergence from Deep Planting Depths and Its Relationship with Coleoptile Length. PLoS ONE 8:e7331.

Narayana Swamy M, Ravikumar C. and Kalmath GP: 2010. Seed germination inhibition test for pregnancy detection in Malnad Gidda Cows. Veterinary World 3: 107-108.

Nirmala G, Veena T, Jyothi M. and Suchitra B: 2008. Effect of estrogen and progesterone on seed germination. Veterinary World 1: 241-242.

Patel OV, Takenouchi N, Takahashi T, Hirako M, Sasaki N. and Domeki I: 1999. Plasma oestrone and oestradiol concentrations throughout gestation in cattle: relationship to stage of gestation and foetal number. Research in Veterinary Science 66: 129-133.

Perumal P: 2014. Pregnancy diagnosis by seed germination inhibition test in mithun (Bos frontalis) cows. Indian Journal of Applied Research 4: 531-532.

Rao Krishna SV. and Veena T: 2009. Evaluation of seed germination test for early detection of pregnancy in cows. Indian Journal of Animal Research 43: 37-40.
Rine M, Hossain M, Akter S, Tarif A, Bari F. and Alam M: 2014. Evaluation of seed germination inhibition test for early detection of pregnancy in cross breed dairy cattle in Bangladesh. Journal of Veterinary Advances 4: 343-349.

Robertson HA. and King GJ: 1979. Conjugated and unconjugated oestrogens in foetal and maternal fluids of the cow throughout pregnancy. Journal of Reproduction and Fertility 55: 463-470.

Shah KD, Nakao T. and Kubota H: 2006. Plasma estrone sulphate (E1S) and estradiol-17 beta (E-2 beta) profiles during pregnancy and their relationship with the relaxation of sacrosciatic ligament, and prediction of calving time in Holstein-Friesian cattle. Animal Reproduction Science 95: 38-53.

Skálová I, Fedorova T. and Baranyiová E: 2017. Seed germination test as a potential pregnancy diagnosis method for domestic cattle. Bulgarian Journal of Agricultural Science 23: 453-461.

Veena Ganesaiah T: 2006. Punyakoti test - An ancient Egyptian test $(2200$ BC) extended to diagnose pregnancy in cattle. p.91-93. Traditional Knowledge Systems of India and Sri Lanka Bangalore. Centre for Indian Knowledge Systems.

Veena T. and Narendranath R: 1993. An ancient Egyptian pregnancy test extended to cattle. Current Science 65: 989-990.

Volkery J, Gottschalk J, Sobiraj A, Wittek T. and Einspanier A: 2012. Progesterone, pregnanediol-3-glucuronide, relaxin and oestrone sulphate concentrations in saliva, milk and urine of female alpacas (Vicugna pacos) and their application in pregnancy diagnosis. Veterinary Record 171: 195.

White J. and Edwards J: 2007. Wheat growth and development. NSW Department of Primary Industries, New South Wales, Australia. 


IOURNAL
OT
ANIMAL
$x$
mant

Yang CJ, Wu LS, Liu SH. and Lin JH: 2004. Monitoring the reproductive status of dairy cows by urinary pregnanediol glucuronide. Asian - Australasian Journal of Animal Sciences 17: 460-466.

Yang CJ, Wu LS, Tseng CM, Chao MJ, Chen PC. and Lin JH: 2003. Urinary estrone sulfate for monitoring pregnancy of dairy cows. Asian-Australasian Journal of Animal Sciences 16: 1254-1260. 